\title{
Editorial
}

\section{When should ACE inhibitors or warfarin be discontinued after myocardial infarction?}

In the current climate of cost containment, discontinuation of medications shown to have efficacy has taken on an urgency that may not appear appropriate to the individual clinician when applied to the individual patient. However, it is often in the patient's best interest to decrease the number of medications prescribed. Following a myocardial infarction, patients are usually discharged on multiple medications including $\beta$ adrenergic receptor blockers, angiotensin converting enzyme (ACE) inhibitors, aspirin, and lipid lowering agents. Polypharmacy is a national health issue that increases the risk of medication errors in formulation or dosage by the prescriber, the pharmacist or the patient, and increases the likelihood of drug interaction. Polypharmacy also decreases patient compliance with the medical regimen. ${ }^{12}$ Cost is a factor for many patients and one that may be underappreciated by physicians. In addition, no drug is without side effects. Discontinuation of a medication removes the possibility of untoward side effects, although at the same time eliminates the possibility of any further therapeutic benefit. It is this risk benefit analysis that must be applied to each patient's situation to determine appropriate pharmacotherapy. Hitherto, no studies have specifically addressed the appropriate timing for discontinuation of ACE inhibitors or warfarin after myocardial infarction, nor are any likely to be performed in the future. Thus these recommendations are necessarily speculative and rely on inferences drawn from the large clinical trials, as well as on of the natural history of ventricular remodelling and thrombus formation.

\section{The case for ACE inhibitors}

The case for ACE inhibition after myocardial infarction appears ironclad. A large meta-analysis established the efficacy of early administration with oral ACE inhibitors in the treatment of unselected patients with acute myocardial infarction. ${ }^{3}$ A $7 \%$ relative reduction in 30 day mortality was observed, with the higher risk subgroups obtaining the greatest benefit. Other studies of patients with symptomatic congestive heart failure or reduced ejection fraction after myocardial infarction have demonstrated a greater relative reduction in mortality $(15-25 \%)$ in the treated groups. ${ }^{4-6}$ The SAVE (survival and ventricular enlargement) trial showed a $19 \%$ relative reduction in total mortality at the end of follow up in patients with ejection fractions lower than $40 \%$ but with no clinical heart failure. The survival advantage was not evident until one year, but the survival curves diverged throughout the remainder of the follow up.

The initial impetus for the use of ACE inhibitors after myocardial infarction derived from animal data demonstrating an attenuation of the remodelling process after large infarctions. Echocardiographic studies confirmed a similar phenomenon in patients. ${ }^{8}$ ACE inhibitors may reduce postinfarction mortality by additional mechanisms. The incidence of myocardial infarction in patients with congestive heart failure was decreased in the treatment group in the SOLVD (studies of left ventricular dysfunction) trial. ${ }^{9}$ Sudden cardiac death was also lower in the ACE inhibition group in a meta-analysis of clinical trials. ${ }^{10}$
The anti-ischaemic effects of this class of drugs have been postulated to be caused by a myriad of systemic and local actions including inhibition of neurohormonal activation, prevention of myocytolysis produced by endogenous angiotensin II, and a decrease in vasoconstriction through the action of increased bradykinin concentrations. ACE inhibitors have recently been suggested to play a more direct role in modulating the atherosclerotic process by increasing endothelium derived relaxing factor (EDRF), prostacyclin, and local PAl-1 production. ${ }^{11}$ Given the salutary effects of ACE inhibition on cardiac events other than remodelling, there is an argument to be made for their long term use in patients at high risk for recurrent cardiac events. However, the side effects of ACE inhibitors can develop at any time during treatment, not only at the initiation of therapy. Hypotension, hyperkalaemia, renal insufficiency, dizziness, syncope, and cough are consistently more frequent in the treatment group in the large clinical trials. ${ }^{9}$ Angioedema may also develop later in the course of ACE inhibitor treatment, requiring discontinuation of the drug.

Predischarge echocardiographic assessment of left ventricular function adds prognostic information beyond that obtained by analysis of clinical demographics or laboratory variables. A low risk subgroup of patients without heart failure, diabetes or uncontrolled hypertension, and with small infarctions and relatively preserved left ventricular function, should have ACE inhibition discontinued at six weeks. This would include those patients with ejection fractions greater than $45 \%$ (as determined by echocardiography) and without anterior or apical wall motion abnormalities. Meta-analysis has demonstrated benefit of ACE inhibitors administered early postinfarction (on day 1) in these patients, but there is little evidence that continuation of the medication beyond six weeks is efficacious. ${ }^{3}$ In the higher risk patient, ACE inhibitors can be justified for up to one year. The echocardiographic substudy of the SAVE trial showed no further attenuation of progressive left ventricular remodelling beyond this time period. The recently published HOPE (heart outcomes prevention evaluation) study demonstrated benefit of ACE inhibition in high risk patients older than 55 years, even in the absence of heart failure and decreased ejection fraction. This patient population had a high incidence of diabetes and those destined to develop diabetes. However, the results support the prolonged use of ACE inhibitors in patients with multiple risk factors for cardiovascular disease. ${ }^{12}$ We would reserve indefinite treatment with ACE inhibitors only in those subgroups who are likely to benefit from the medication for other reasons: patients with symptomatic heart failure, patients with diabetes, particularly with nephropathy, and hypertensive patients who have achieved normotensive control on these agents. Use of ACE inhibitors should never preclude treatment with $\beta$ blockers in postinfarction patients in whom long term benefit has been well established. 


\section{Left ventricular thrombus formation}

While ventricular remodelling is a universal process after myocardial infarction, left ventricular thrombus formation is not. The incidence of left ventricular thrombus detected by routine echocardiography appears to be declining in recent studies compared to historical controls. While previously reported to occur in up to $25 \%$ of patients with acute Q wave myocardial infarctions and $40 \%$ of patients with anterior infarctions, one recent study found an incidence as low as $3.7 \%$ in patients who had received thrombolytic agents and early anticoagulation. ${ }^{13}$ Other studies have shown a less dramatic decrease in the prevalence of ventricular thrombus in the thrombolytic era, but a definite effect of combination treatment with unfractionated or low molecular weight heparin in addition to thrombolysis in reducing the appearance of thrombus on echocardiography. ${ }^{14}{ }^{15}$ Attenuation of ventricular dilatation and aneurysm formation with ACE inhibition may also play a role in the decreased incidence. Many of the studies of left ventricular thrombus have included small numbers of patients. A meta-analysis of patients with anterior myocardial infarction supported the conclusion that anticoagulation did reduce the risk of thrombus formation and embolisation. Furthermore, the vast majority of left ventricular thrombi visualised on echocardiography resolved within three months on anticoagulation. ${ }^{16}$

Postinfarction patients at high risk for adverse cardiovascular events may be identified on a predischarge echocardiogram by their increased left ventricular volume. Patients with anterior myocardial infarction and significant wall motion abnormality or aneurysm and those with definite thrombus should be anticoagulated. There is no evidence that anticoagulation longer than three months will decrease the risk of embolic events, and the risks of chronic anticoagulation with warfarin are well known. Two groups of patients should be considered for longer term treatment. Both the SOLVD ${ }^{17}$ and SAVE ${ }^{18}$ trials demonstrated benefit of chronic anticoagulation in patients with ejection fractions of $35 \%$ or lower. It is also difficult to justify discontinuing anticoagulation in a patient with a "high risk" thrombus. The protruding, mobile thrombus adjacent to an area of hyperkinesis has a high likelihood of embolisation. ${ }^{19}$ A randomised study of anticoagulation in patients with a high risk thrombus will never be done because of ethical considerations. A reasonable strategy is to repeat an echocardiogram at three months after myocardial infarction and continue warfarin only in those patients with a high risk thrombus, left ventricular aneurysm or severely reduced ejection fraction.

Echocardiography provides important information that allows appropriate adjustment of medication after myocardial infarction beyond the clinically described high risk group of patients with overt heart failure after anterior infarctions and those with diabetes, and postinfarction ischaemia. Thus, ACE inhibitors can be safely discontinued at six weeks, and warfarin at three months, in many patients. Patients who are likely to benefit from more prolonged treatment can be identified as those with high risk clinical characteristics, including age over 55 years and a history of diabetes, hyperlipidaemia or hypertension. Simi- larly, those patients identified echocardiographically as having severely reduced left ventricular function (left ventricular ejection fractions of less than $35 \%$ ), extensive wall motion abnormalities or thrombus may benefit from prolonged treatment with warfarin and ACE inhibitors. This strategy allows the directing of resources to patients most likely to benefit, and avoidance of serious side effects and costs with the others.

Hospital of the University of Pennsylvania,

SUSAN E WIEGERS MARTIN ST JOHN SUTTON

3400 Spruce Street, 9 E Gates Pavilion,

Philadelphia, PA 19104, US

email:suttonm@mail.med.upenn.edu

1 Bjerrum L, Sogaard J, Hallas J, et al. Polypharmacy: correlations with sex, age and drug regimen. A prescription database study. Eur 7 Clin Pharmacol 1998;54:197-202

2 Fincke BG, Miller DR, Spiro AR. The interaction of patient perception of overmedication with drug compliance and side effects. F Gen Intern Med 1998;13:182-5.

3 Anon. Indications for ACE inhibitors in the early treatment of acute myocardial infarction: systematic overview of individual data from 100,000 myocardial infarction: systematic overview of individual data from 100,000 patients in randomized trials. ACE inhibito
rative group. Circulation 1998;97:2202-12.

rative group. Circulation 1998;97:2202-12.
4 GISSI-3 Investigators. GISSI-3: effects of lisinopril and transdermal glyceryl trinitrate singly and together on 6 -week mortality and ventricular function after acute myocardial infarction. Gruppo Italiano per lo studio della sopravvivenza nell'infarto miocardico. Lancet 1994;343:1115-22.

5 AIRE Study Investigators. Effect of ramipril on mortality and morbidity of survivors of acute myocardial infarction with clinical evidence of heart failure. The acute infarction ramipril efficacy (AIRE) study investigators. Lancet 1993;342:821-8.

6 Kober L, Torp-Pedersen C, Carlsen JE, et al. A clinical trial of the angiotensin-converting-enzyme inhibitor trandolapril in patients with left
ventricular dysfunction after myocardial infarction. Trandolapril cardiac evaluation (TRACE) study group. N Engl f Med 1995;333:1670-6.

7 Pfeffer MA, Braunwald E, Moye LA, et al. Effect of captopril on mortality and morbidity in patients with left ventricular dysfunction after myocardial infarction. Results of the survival and ventricular enlargement trial. The infarction. Results of the survival and ventricular en
SAVE Investigators. N Engl 7 Med 1992;327:669-77.

8 St John Sutton M, Pfeffer MA, Moye L, et al. Cardiovascular death and left ventricular remodeling two years after myocardial infarction: baseline predictors and impact of long-term use of captopril: information from the survival and ventricular enlargement (SAVE) trial. Circulation 1997;96:3294-

9 Anon. Effect of enalapril on survival in patients with reduced left ventricular ejection fractions and congestive heart failure. N Engl f Med 1991;325:293302 .

10 Domanski MJ, Exner DV, Borkowf CB, et al. Effect of angiotensin converting enzyme inhibition on sudden cardiac death in patients following acute myocardial infarction. A meta-analysis of randomized clinical trials. $7 \mathrm{Am}$ Coll Cardiol 1999;33:598-604.

11 Brown N, Vaughan D. Angiotensin-converting enzyme inhibitors. Circulation 1998;97:1411-1420.

12 The Heart Outcomes Prevention Evaluation Study Investigators. Effects of an angiotensin-converting-enzyme inhibitor, ramipril, on cardiovascular events in high-risk patients. N Engl f Med 2000;342:145-53.

13 Greaves SC, Zhi G, Lee RT, et al. Incidence and natural history of left ventricular thrombus following anterior wall acute myocardial infarction. Am 7 Cardiol 1997;80:442-8.

14 Vecchio C, Chiaarelli F, Lupi G, et al. Left ventricular thrombus in anterior acute myocardial infarction after thrombolysis. F Am Coll Cardiol 1991;84: 512-9.

15 Kontny F, Dale J, Abildgaard U, et al. Randomized trial of low molecular weight heparin in prevention of left ventricular thrombus formation and arterial embolism after acute anterior myocardial infarction: the Fragmin in acute myocardial infarction (FRAMI) study. If Am Coll Cardiol 1997;30: 962-9.

16 Kupper AJ, Verheugt FW, Peels $\mathrm{CH}$, et al. Left ventricular thrombus incidence and behavior studied by serial two-dimensional echocardiography in acute anterior myocardial infarction: left ventricular wall motion, systemic embolism and oral anticoagulation. F Am Coll Cardiol 1989;13: $1514-20$.

17 Al-Khadra A, Salem D, Rand W, et al. Warfarin anticoagulation and survival: a cohort analysis from the studies of left ventricular dysfunction. $₹ \mathrm{Am}$ Coll Cardiol 1998:31:749-53.

18 Loh E, St John Sutton M, Wun C, et al. Ventricular dysfunction and the risk of stroke after myocardial infarction. N Engl f Med 1997;336:251-7.

19 Jugdutt B, Sivaram C. Prospective two-dimensional echocardiographic evaluation of left ventricular thrombus and embolism after acute myocardial infarction. $7 \mathrm{Am}$ Coll Cardiol 1989;13:554-64. 